\title{
A practical approach to prescribing antiplatelet therapy in patients with acute coronary syndromes
}

\author{
Mohammad Alkhalil DPhil, Michał Kuzemczak MD PhD, Alan Bell MD, Sol Stern MD, Michelle Welsford MD, \\ Warren J. Cantor MD, Shaun G. Goodman MD
}

Cite as: CMAJ 2022 February 14;194:E205-15. doi: 10.1503/cmaj.202863

A fter an acute coronary syndrome, as many as 1 in 5 patients will have a second ischemic event within 5 years. ${ }^{1,2}$ Residual risk is related to several factors that may be mitigated by pharmacologic and nonpharmacologic interventions. ${ }^{2}$ Antiplatelet therapy is a cornerstone in the management of acute coronary syndrome. ${ }^{1,3}$ Acetylsalicylic acid (ASA) - a cyclooxygenase-1 inhibitor - was introduced as an effective treatment for myocardial infarction almost 5 decades ago and remains the most widely used antiplatelet therapy. ${ }^{4,5}$ Although ASA is effective in reducing mortality rates, ${ }^{6}$ combining ASA with a second antiplatelet agent, a $\mathrm{P} 2 \mathrm{Y}_{12}$ receptor inhibitor (known as dual antiplatelet therapy [DAPT]) provides additional benefit and is now the preferred initial strategy for acute coronary syndromes over ASA alone. ${ }^{7}$

We review emerging evidence regarding the use of antiplatelet therapy in acute coronary syndromes, as well as updates to the Canadian and European Society of Cardiology guidelines that highlight adjustments in the choice and duration of antiplatelet therapy, in addition to ASA (Box 1). We particularly focus on strategies to reduce bleeding risk after percutaneous coronary intervention $(\mathrm{PCl}), 3,8$

\section{What are the options for oral antiplatelet therapy?}

Clopidogrel is a second-generation thienopyridine that has a better safety profile than ticlopidine, a first-generation thienopyridine. ${ }^{9}$ Clopidogrel reduces ischemic events by almost $20 \%$ when added to ASA for patients presenting with acute coronary syndromes,

\section{Box 1: Literature search}

We conducted a targeted, nonsystematic MEDLINE search from its inception until August 2021 using the terms "antiplatelet," "clopidogrel," "prasugrel," "ticagrelor," "acute coronary syndrome," "myocardial infarction" or "bleeding." We limited the search to articles in English and focused on randomized clinical trials or systematic reviews, although we did not apply any restriction on study type.

\section{Key points}

- Prasugrel and ticagrelor are $\mathrm{P} 2 \mathrm{Y}_{12}$ inhibitors that are more effective than clopidogrel at preventing ischemic events in patients presenting with acute coronary syndrome, although they cause more bleeding.

- Dual antiplatelet therapy (acetylsalicylic acid and a P2Y ${ }_{12}$ inhibitor) is indicated for patients with acute coronary syndrome, usually for at least 1 year.

- The type and duration of dual antiplatelet therapy is determined by a patient's risk of recurrent ischemia and major bleeding.

- Most patients with atrial fibrillation and acute coronary syndrome should be treated with a direct oral anticoagulant and clopidogrel for 12 months; acetylsalicylic acid should be added for up to 1 month after percutaneous coronary intervention.

with or without ST-segment elevation. ${ }^{7,10,11}$ It irreversibly antagonizes the receptor for platelet adenosine diphosphate (ADP)$\mathrm{P} 2 \mathrm{Y}_{12}$. The use of clopidogrel may be associated with gastrointestinal symptoms and skin rashes.

Prasugrel and ticagrelor are more potent $P 2 Y_{12}$ inhibitors than clopidogrel. Prasugrel is a third-generation thienopyridine that exerts its antiplatelet properties by irreversibly antagonizing the $A D P-P_{2} Y_{12}$ receptor; similar to clopidogrel, it requires hepatic conversion to its active metabolites. Ticagrelor is part of the cyclopentyltriazolopyrimidine family and does not require hepatic conversion to its active metabolites before reversibly inhibiting the ADP- $\mathrm{P}_{2} \mathrm{Y}_{12}$ receptor. Ticagrelor may cause shortness of breath or increased levels of uric acid, which leads to gout.

Both prasugrel and ticagrelor provide faster and more consistent inhibition of platelet aggregation and are associated with a further $15 \%-20 \%$ relative risk reduction of ischemic events compared with clopidogrel. ${ }^{12,13}$ Ticagrelor also reduces cardiovascular and all-cause mortality rates. ${ }^{12}$ Although ticagrelor and prasugrel are associated with greater bleeding risk than clopidogrel, both are recommended over clopidogrel in patients with low bleeding risk. Recently, the 
ISAR-REACT-5 (Intracoronary Stenting and Antithrombotic Regimen 5) study reported fewer ischemic events associated with prasugrel, with no difference in the incidence of major bleeding, when compared with ticagrelor. ${ }^{14}$ However, a number of uncertainties preclude definitively recommending one drug over the other. ${ }^{15}$ Prasugrel is not recommended in patients older than 75 years of age and in those with a body weight less than $60 \mathrm{~kg}$ because of an increased risk of fatal and intracranial bleeding. ${ }^{13} \mathrm{~A}$ recent meta-analysis summarized the relative differences in ischemic and bleeding risks among the 3 different $P 2 Y_{12}$ inhibitors (Table 1 ). ${ }^{16}$

Clopidogrel is currently recommended for patients with acute coronary syndromes who are at high bleeding risk or those who cannot take a potent $\mathrm{P}_{2} \mathrm{Y}_{12}$ inhibitor because of adverse effects or cost. ${ }^{3,8}$ Guidelines recommend treatment with clopidogrel as the initial antiplatelet drug for patients with ST-segment elevation myocardial infarction who are treated with fibrinolytic therapy. However, results of the TREAT (Ticagrelor in Patients With ST-Elevation Myocardial Infarction Treated With Pharmacological Thrombolysis) study indicated that switching from clopidogrel to ticagrelor within 24 hours did not lead to an increase in major bleeding in the first 30 days postlysis, compared with continuing clopidogrel. ${ }^{17}$

Current guidelines recommend the use of DAPT after an acute coronary syndrome, irrespective of the revascularization strategy, including for medically managed patients and those who undergo coronary artery bypass grafts. ${ }^{3,18} \mathrm{~A}$ subgroup analysis of 7985 patients from the CURE (Clopidogrel in Unstable Angina to Prevent Recurrent Events) trial who did not undergo revascularization after randomization showed that adding clopidogrel to ASA reduced ischemic events by an absolute $1.9 \%$ after 12 months of follow-up, compared with placebo and ASA. ${ }^{7}$ Ticagrelor provides consistent benefit over clopidogrel in reducing ischemic events, irrespective of revascularization strategy. ${ }^{12,19}$

For patients with non-ST segment elevation acute coronary syndrome, (NSTACS) the timing of coronary angiography is a factor in the choice of antiplatelet treatment. For patients scheduled for coronary angiography within 24 hours of presentation, there is debate regarding whether patients should be preloaded with a potent $P 2 Y_{12}$ inhibitor. Preloading with prasugrel or ticagrelor did not reduce ischemic events in patients with NSTACS scheduled for early coronary angiography. ${ }^{20,21}$ Thus, the European guidelines discourage routine preloading of $\mathrm{P}_{2} \mathrm{Y}_{12}$ inhibitors in patients with NSTACS who are planned for an early coronary angiogram and suggest starting DAPT once the need for angioplasty or stents is confirmed. ${ }^{3}$ This does not apply for patients presenting with ST-segment elevation myocardial infarction in whom preloading with DAPT is recommended. Preloading should also be considered in non- $\mathrm{PCl}$ centres because coronary angiography may be delayed. Further, given that, in Canada, most patients with NSTACS do not routinely undergo angiography within the first 24 hours of presentation, preloading with DAPT in patients with moderate-to-high risk of ischemia is reasonable. ${ }^{8}$ If there is suspicion of left main disease or possible aortic dissection, DAPT should not be given. ${ }^{3}$

\section{How long should dual antiplatelet therapy be continued?}

Current guidelines recommend DAPT for 1 year after an acute coronary syndrome, particularly in patients with heightened ischemic risk (Box 2 and Figure 1). ${ }^{3,8}$ However, patients at high risk of bleeding may be considered for shorter treatment duration. Two large randomized controlled trials evaluated extending the duration of DAPT beyond 12 months, using ticagrelor in patients with a history of myocardial infarction in the PEGASUS-TIMI 54 (Prevention with Ticagrelor of Secondary Thrombotic Events in High-Risk Patients with Prior Acute Coronary Syndrome - Thrombolysis In Myocardial Infarction 54) trial, and using clopidogrel or prasugrel in stable patients and patients with acute coronary syndromes who received a $\mathrm{PCl}$ in the Dual Antiplatelet Therapy trial. ${ }^{22,23}$ Both trials reported significant relative reductions in ischemic events of $15 \%$ $30 \%$, but these benefits were offset by a significant increase in major bleeding. ${ }^{22,23} \mathrm{~A}$ recent meta-analysis confirmed these findings; DAPT extension beyond 12 months was associated with a $32 \%$ reduction in myocardial infarction and a $63 \%$ increase in major bleeding among patients who received a $\mathrm{PCl}^{24}$

\section{Table 1: Relative difference in ischemic and bleeding risks ${ }^{\star}$ among different $\mathbf{P}_{2} \mathbf{Y}_{12}$ inhibitors ${ }^{16}$}

\begin{tabular}{|c|c|c|c|}
\hline Drugs compared & $\begin{array}{l}\text { No. of studies } \\
\text { (no. of patients) }\end{array}$ & Ischemic risk & Bleeding risk \\
\hline $\begin{array}{l}\text { Ticagrelor v. } \\
\text { clopidogrel }\end{array}$ & $\begin{array}{c}6 \text { RCTs } \\
(21828) \dagger\end{array}$ & $\begin{array}{l}\text { Ticagrelor associated with } 18 \% \text { reduction in } \\
\text { cardiovascular mortality, } 28 \% \text { reduction in stent } \\
\text { thrombosis and no difference in } \mathrm{MI}\end{array}$ & $\begin{array}{l}\text { Ticagrelor associated with } 27 \% \\
\text { increase in major bleeding }\end{array}$ \\
\hline $\begin{array}{l}\text { Prasugrel v. } \\
\text { clopidogrel }\end{array}$ & $\begin{array}{l}4 \text { RCTs } \\
(25740)\end{array}$ & $\begin{array}{l}\text { Prasugrel associated with } 10 \% \text { reduction in } \\
\text { cardiovascular mortality ( } 95 \% \mathrm{Cl} 0.80-1.01), 50 \% \\
\text { reduction in stent thrombosis and } 19 \% \\
\text { reduction in } \mathrm{MI}\end{array}$ & $\begin{array}{l}\text { Prasugrel associated with } 26 \% \\
\text { increase in major bleeding }\end{array}$ \\
\hline Prasugrel v. ticagrelor & $\begin{array}{l}2 \text { RCTs } \\
(5248)\end{array}$ & $\begin{array}{l}\text { Prasugrel associated with } 32 \% \text { reduction in stent } \\
\text { thrombosis and no difference in cardiovascular } \\
\text { mortality or } \mathrm{MI}\end{array}$ & No difference in major bleeding \\
\hline
\end{tabular}




\section{Box 2: Features of patients at high risk of ischemic} events

- Previous stent thrombosis on adequate antiplatelet therapy

- Stenting of the last remaining patent coronary artery

- Diffuse multivessel disease, especially in patients with diabetes

- Chronic kidney disease (i.e., creatinine clearance $<60 \mathrm{~mL} / \mathrm{min}$ )

- At least 3 stents implanted

- At least 3 lesions treated

- Bifurcation with 2 stents implanted

- Total stented length greater than $60 \mathrm{~mm}$

- Treatment of a chronic total occlusion

- History of ST-segment elevation myocardial infarction

Improvements in stent design and increased recognition of the importance of preventing bleeding led researchers to evaluate shorter durations of DAPT; the findings of noninferiority studies of stable patients and patients with acute coronary syndrome after
$\mathrm{PCl}$ have supported this approach. ${ }^{25}$ Meta-analyses found comparable incidences of stent thrombosis and adverse ischemic events; however, myocardial infarction was more frequent in the shortened DAPT group. ${ }^{25}$ Recently, the MASTER-DAPT (Management of High Bleeding Risk Patients Post Bioresorbable Polymer Coated Stent Implantation With an Abbreviated Versus Prolonged DAPT Regimen) study randomized 4434 patients at high risk of bleeding who were free of cardiovascular events at 1 month after $\mathrm{PCl}$ to receive 1 month of DAPT or standard DAPT of at least 3 months after $\mathrm{PCl} .{ }^{26}$ The measure of adverse clinical events (defined as a composite of death from any cause, myocardial infarction, stroke or major bleeding) was comparable between the 2 groups. ${ }^{26}$ Importantly, the incidence of major bleeding or clinically relevant, nonmajor bleeding was significantly lower in the shorter treatment group (6.5\% v. 9.4\%, $p<0.001){ }^{26}$

The use of a single antiplatelet agent was recently tested in an open-label, multicentre randomized study of 5438 patients who had completed 6-18 months of DAPT without any clinical events, compared with ASA alone. Over a mean follow-up of 24 months, clopidogrel reduced the composite outcome of all-cause death,

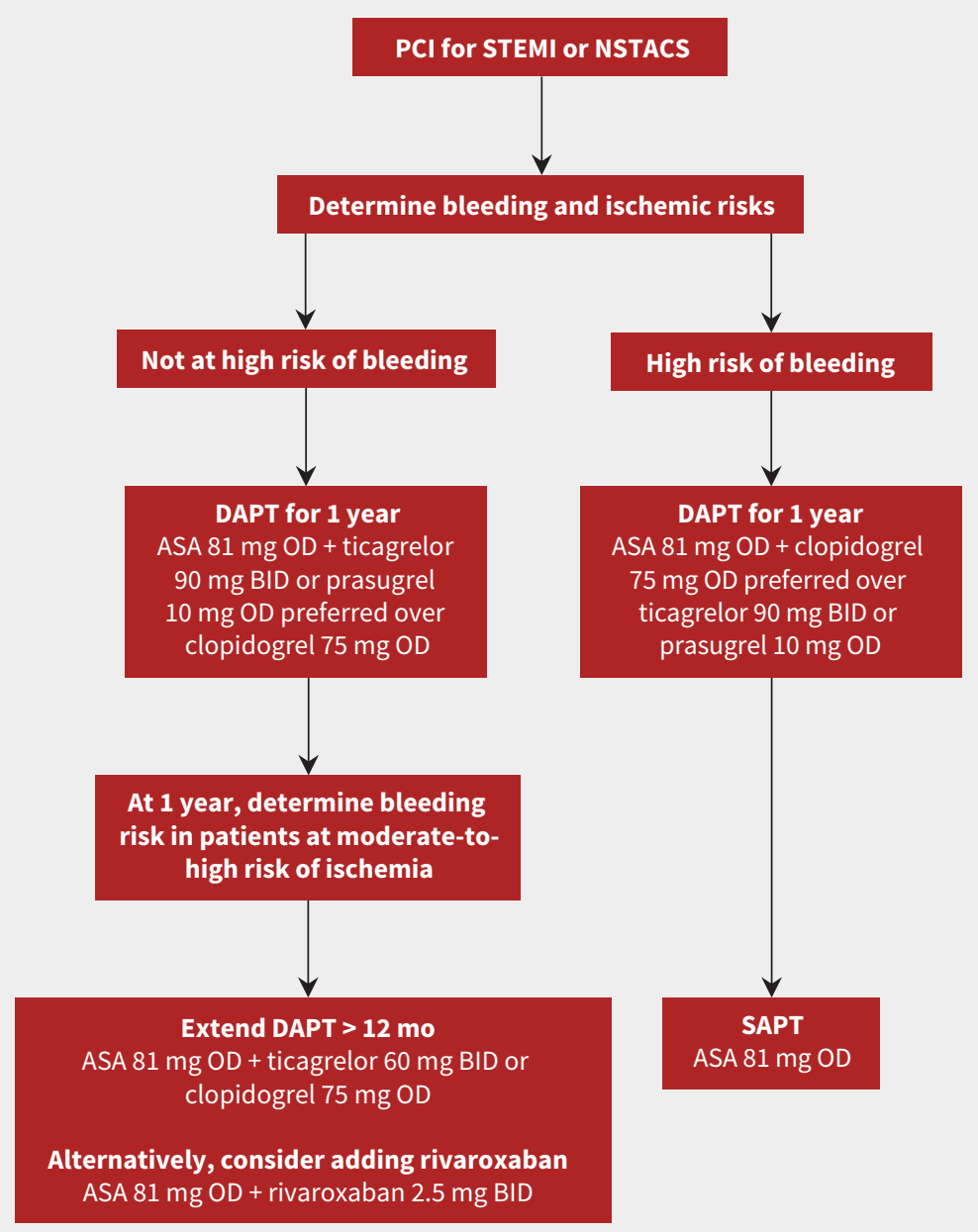

Figure 1: Antiplatelet recommendations in patients with acute coronary syndrome (ACS) who underwent percutaneous coronary intervention (PCI). Note: ASA = acetylsalicylic acid, BID = twice daily, DAPT = dual antiplatelet therapy, NSTACS = non-ST segment elevation acute coronary syndrome, $\mathrm{OD}=$ once daily, SAPT = single antiplatelet therapy, STEMI = ST-segment elevation myocardial infarction. 
myocardial infarction, stroke, readmission because of an acute coronary syndrome and major bleeding events (5.7\% v. 7.7\%; hazard ratio $[\mathrm{HR}] 0.73,95 \%$ confidence interval $[\mathrm{Cl}]$ 0.59-0.90). ${ }^{27}$ Both ischemic (3.7\% v. 5.5\%; HR 0.68, 95\% Cl 0.52-0.87) and any bleeding events ( $2.3 \%$ v. $3.3 \%$; HR $0.70,95 \% \mathrm{Cl} 0.51-0.98)$ were reduced with the use of clopidogrel.

Other strategies for extended secondary prevention include the use of a low dose of oral anticoagulant in combination with ASA. This has been used in the COMPASS (Cardiovascular Outcomes for People Using Anticoagulation Strategies) trial for patients with stable atherosclerotic cardiovascular disease, including previous myocardial infarction, cerebrovascular and peripheral artery disease. ${ }^{28}$ Compared with ASA alone, rivaroxaban ( $2.5 \mathrm{mg}$ twice daily) and ASA reduced ischemic events by an absolute $1.3 \%$, but was associated with more major bleeding (3.1\% v. $1.9 \%){ }^{28}$

\section{How is bleeding risk assessed and managed?}

Models have been developed to quantify bleeding risk; ${ }^{1,29}$ they include clinical and biomarker variables and have moderate-to-good accuracy (Table 2). The DAPT, PRECISE-DAPT and PRAISE models are accessible online. In a meta-analysis of 88563 patients, the DAPT score consistently identified patients at high risk of bleeding and ischemia in different cohorts of patients. ${ }^{39}$ Similarly, the PRECISEDAPT model effectively identified patients who were not suitable for extended DAPT and were likely to be at risk of bleeding without a decrease in ischemic events. ${ }^{35}$ The PRECISE-DAPT model was also validated in cohorts of patients with acute coronary syndromes who underwent $\mathrm{PCl}$ and were treated with potent $\mathrm{P}_{2} \mathrm{Y}_{12}$ inhibitors, and showed moderate accuracy in predicting future bleeding risk. ${ }^{40}$

The PRAISE model used machine learning to predict bleeding, ischemic risk and all-cause deaths. ${ }^{38}$ The model was derived from 2 cohorts of patients with acute coronary syndromes who were treated with clopidogrel or more potent $\mathrm{P} 2 \mathrm{Y}_{12}$ inhibitors. It accurately predicted major bleeding, as well as acute $\mathrm{MI}$ and all-cause mortality. Importantly, machine learning risk-scoring models need to be tested in randomized controlled trials to assess their impact on clinical outcomes.

Strategies to reduce bleeding risk at the time of $\mathrm{PCl}$ include the use of radial access (preferable to accessing a more central artery), using fluoroscopy or ultrasonography guidance to access the common femoral artery ${ }^{41}$ and selecting the right stent platform that is indicated for patients with high bleeding risk or is considered safe for early discontinuation of DAPT. ${ }^{26,42}$ Bleeding risk can also be reduced with the use of clopidogrel rather than prasugrel or ticagrelor in patients who are at high risk. ${ }^{41}$ After hospital discharge, several strategies should be used to reduce bleeding risk (Box 3 ).

The management of acute bleeding events is discussed elsewhere. ${ }^{44}$ Current guidelines categorize patients according to the type of bleeding event and recommend management according to severity (Table 3). ${ }^{18}$ Reversal agents are currently available for some oral anticoagulants, and antidotes to $\mathrm{P}_{2} \mathrm{Y}_{12}$ inhibitors are being developed. ${ }^{45} \mathrm{~A}$ ticagrelor reversal agent, bentracimab (PB2452), is a human monoclonal antibody that provides immediate and sustained reversal of the antiplatelet effects of ticagrelor in healthy volunteers. ${ }^{45}$ It is currently being studied in the REVERSE-IT (Rapid
Box 3: Strategies to reduce bleeding risk following hospital discharge

- Shorter duration of dual antiplatelet therapy

- Use of clopidogrel rather than ticagrelor or prasugrel

- Avoidance of nonsteroidal anti-inflammatory drugs

- Optimal blood pressure management

- Abstinence from alcohol

- Use of mobility aids, when appropriate

- Use of proton pump inhibitor for all patients after percutaneous coronary intervention, as recommended by current guidelines. ${ }^{18}$

- Screening and eradication of Helicobacter pylori (a large randomized controlled trial is underway to assess this strategy in patients with acute myocardial infarction $)^{43}$

- Correction of anemia to reduce the impact of bleeding

and Sustained Reversal of Ticagrelor-Intervention Trial) trial (NCT04286438). Management of bleeding events in patients on DAPT can be challenging and involvement of specialists should be considered (Box 4).

\section{Can treatment with acetylsalicylic acid be stopped early?}

Early stopping of ASA while maintaining $P 2 Y_{12}$ inhibition has recently been tested, based on experimental data that suggested the synergistic effect of inhibiting cyclooxygenase-1 with ASA and $P 2 Y_{12}$ inhibitors is less relevant in the presence of potent $P 2 Y_{12}$ inhibitors. ${ }^{46} \mathrm{~A}$ recent meta-analysis of data from 16898 patients with acute coronary syndromes showed that $\mathrm{P}_{2} \mathrm{Y}_{12}$ inhibitor monotherapy after 1-3 months of DAPT reduced bleeding events by $50 \%$ with no significant increase in ischemic events, compared with 12 months of DAPT. ${ }^{24,47}$ A recent meta-analysis included individual data from 24096 patients enrolled in 6 randomized trials and highlighted the superiority of $\mathrm{P}_{2} \mathrm{Y}_{12}$ inhibitor monotherapy over DAPT in reducing ischemic events in women, and the reduction of

Box 4: Indications for referral to cardiologist and other specialist regarding antiplatelet treatment

- Bleeding events in patients within 1 year of acute coronary syndrome; advice from a cardiologist about resumption or discontinuation of a second antiplatelet agent should be sought early, and consultation with a gastroenterologist should also be considered, if indicated

- New onset atrial fibrillation

- Patients with planned noncardiac surgery (particularly major surgeries)

- Patients with chronic bleeding diatheses, such as hemophilia or severe liver disease; consultation with a hematologist should be considered

- Patients who develop thrombocytopenia; consultation with a hematologist should be considered

- Patients who develop a cerebrovascular event; consultation with a neurologist should be considered 
Table 2 (part 1 of 2): Models to estimate bleeding risk

\begin{tabular}{|c|c|c|c|}
\hline Model & Derived population & Score variables & Score description \\
\hline CRUSADE ${ }^{30}$ & $\begin{array}{l}71277 \text { community- } \\
\text { treated patients with } \\
\text { NSTEMI }\end{array}$ & $\begin{array}{l}\text { Hematocrit, creatinine } \\
\text { clearance, baseline heart rate, } \\
\text { baseline systolic blood } \\
\text { pressure, female sex, signs of } \\
\text { CHF on presentation, previous } \\
\text { vascular disease and diabetes } \\
\text { mellitus }\end{array}$ & $\begin{array}{l}\text { Each independent variable was } \\
\text { assigned weighted integers } \\
\text { according to its coefficient value in } \\
\text { the regression model. The sum of the } \\
\text { weighted integers (range } 1 \text { to } \\
100 \text { points) estimates the risk of } \\
\text { in-hospital major bleeding, with a } \\
\text { curvilinear relation between } \\
\text { CRUSADE bleeding score and } \\
\text { predicted probabilities of major } \\
\text { bleeding }\end{array}$ \\
\hline ACUITY ${ }^{31}$ & $\begin{array}{l}17421 \text { patients with } \\
\text { ACS (UA, NSTEMI and } \\
\text { STEMI) }\end{array}$ & $\begin{array}{l}\text { Age, female sex, serum } \\
\text { creatinine, white blood cell } \\
\text { count, anemia, NSTEMI, } \\
\text { STEMI and the use of heparin } \\
\text { plus glycoprotein Ilb/IIla } \\
\text { inhibitor (rather than } \\
\text { bivalirudin alone) }\end{array}$ & $\begin{array}{l}\text { Each independent variable was } \\
\text { assigned weighted integers } \\
\text { according to its coefficient value in } \\
\text { the regression model. The sum of the } \\
\text { weighted integers (range } 1 \text { to } \\
52 \text { points) estimates the risk of } \\
30 \text {-day non-CABG major bleeding, } \\
\text { with curvilinear relation between } \\
\text { ACUITY bleeding score and predicted } \\
\text { probabilities of bleeding }\end{array}$ \\
\hline
\end{tabular}

$\begin{array}{ll}\mathrm{REACH}^{32} & 64589 \text { at risk of CAD } \\ \text { or with stable CAD }\end{array}$

$\mathrm{DAPT}^{33 *}$
Age, peripheral arterial disease, CHF, diabetes, hypertension, smoking, antiplatelets, oral anticoagulants, hypercholesterolemia
Each factor was assigned a single point, except for CHF, hypertension, smoking and non-ASA antiplatelet therapy, which were assigned 2 points. Oral anticoagulation or DAPT were assigned 4 points. A score $>10$ was associated with 6 -fold increase in risk of serious bleeding over 2 years
Age, cigarette smoking, diabetes mellitus, $\mathrm{Ml}$ at presentation, previous $\mathrm{PCl}$ or previous MI, paclitaxel-eluting stent, stent diameter $<3 \mathrm{~mm}$, $\mathrm{CHF}$ or $\mathrm{LVEF}<30 \%$, and vein graft stent
Each variable was assigned a single point except for age $(65$ to $<75 \mathrm{yr}$ and $\geq 75 \mathrm{yr}$, for which patients were assigned -1 or -2 , respectively). Those with CHF, LVEF or vein graft stent were assigned 2 points. Total scores ranged from -2 to 10 , and those with scores $\geq 2$ were considered high risk and extended DAPT was recommended. Patients with low scores $(<2)$ were considered low risk and extended DAPT was not recommended

An integer-based risk score was developed for major bleeding (and ischemic events) at 2 years by assigning each variable a score of 2 , except for anemia (score of 3 ) and age (higher score proportional to older patients). The score ranges from 0 to 14 and $\geq 8$ is considered high bleeding risk
Patients who died within 48 hours were excluded and early bleeding events may be underestimated. Patients on oral anticoagulation were excluded; similarly those with previous bleeding events or bleeding disorders were not included. CRUSADE is designed to predict in-hospital bleeding events

Posthoc analysis of patients included in 2 RCTs. Potential variables of interest were not available to be incorporated in the model. Potent P2Y 12 inhibitors were not studied

The definition of serious bleeding used for the analyses was either a hemorrhagic stroke or bleeding leading to both hospitalization and transfusion. This may underestimate the rate of major bleeding events. Data regarding potent $P 2 Y_{12}$ inhibitors were limited. The exposure to oral anticoagulation was extrapolated and did not account for potential changes over study follow-up

DAPT score showed moderate accuracy in the derivation and validation cohort. It is designed to inform the duration of DAPT rather than predicting future bleeding events

Most patients were treated with clopidogrel, which limits generalizability to potent $P 2 Y_{12}$ inhibitors. Duration of DAPT was not randomized and decision to stop antiplatelet was according to the clinician's discretion 
Table 2 (part 2 of 2): Models to estimate bleeding risk

\begin{tabular}{|c|c|}
\hline Model & Derived population \\
\hline $\begin{array}{l}\text { PRECISE- } \\
\text { DAPT }^{35 *}\end{array}$ & $\begin{array}{l}14963 \text { patients } \\
\text { treated with DAPT } \\
\text { after PCI were } \\
\text { pooled from } 8 \text { RCTs } \\
\text { with independent } \\
\text { adjudication of } \\
\text { events }\end{array}$ \\
\hline
\end{tabular}

\section{Score variables}

Age, creatinine clearance, hemoglobin, white blood cell count and previous spontaneous bleeding.
ARC-HBR trade-off mode $^{36,37}$

PRAISE $^{38 *}$
6641 patients who underwent $\mathrm{PCl}$ and were identified as HBR were pooled from 6 studies

\author{
Age, hemoglobin, renal \\ dysfunction, liver disease, \\ cancer, planned major \\ surgery, COPD, current \\ smoker, complex $\mathrm{PCl}$ \\ procedure, oral \\ anticoagulation at discharge
}

Age, sex, diabetes, hypertension, hyperlipidemia, PAD, eGFR, previous MI, previous $\mathrm{PCl}$, previous $\mathrm{CABG}$, previous stroke, previous bleeding, malignancy, STEMI, LVEF, multivessel disease, complete revascularization, vascular access, DES and treatment with BBs, ACE inhibitors, ARBs, statins, oral anticoagulation and PPIs

Score description

Limitations

Independent predictors of bleeding events that were identified in the multivariate regression model were assigned points based on the magnitude of association of each predictor with bleeding. A score $\geq 25$ is considered $\mathrm{HBR}$, and extended DAPT has been associated with increased bleeding in this group, unlike patients with low scores. PRECISE-DAPT showed improved integrated discrimination and reclassification performance compared with the PARIS score

Variables were classified as a major or minor criterion for HBR. Major criterion is considered to confer risk of major bleeding of $\geq 4 \%$ or $\geq 1 \%$ intracranial hemorrhage at 1 year. This score outperformed PARIS and PRECISE-DAPT (alternative model without white blood cell count)

Four machine learning models that used different classifiers were developed to predict occurrence of all-cause death, recurrent $\mathrm{MI}$ and major bleeding 1 year after discharge. Each model's performance was assessed using a range of learning metrics and the best performing model was selected. When calculated, PRAISE provides 3 outcomes of the calculated score for death, MI, and major bleeding.
The accuracy of the model in the validation cohort ranged from moderate to good. Frailty was not included as part of the risk model. Most patients were treated with clopidogrel and those on oral anticoagulation were excluded. Prediction of bleeding events in patients on prasugrel was poor

The criteria used were modified from those proposed in the ARC consensus to allow the use of available data. Infrequent, but wellrecognized, predictors were pooled as a single variable in the model (i.e., liver disease, cancer and planned surgery)

It was not possible to fully compare PRAISE with PARIS or PRECISE-DAPT given the insufficient clinical data. Although PRAISE has been prospectively validated in external cohorts, it has not been used in RCTs to aid decision-making of DAPT duration

Note: $\mathrm{ACE}=$ angiotensin-converting enzyme, $\mathrm{ACS}=$ acute coronary syndrome, $\mathrm{ACUITY}=$ Acute Catheterization and Urgent Intervention Triage Strategy, $\mathrm{ARB}=$ angiotensin-receptor blocker, $\mathrm{ARC}=\mathrm{Academic}$ Research Consortium, $\mathrm{ASA}=$ acetylsalicylic acid, $\mathrm{BB}=$ beta-blocker, $\mathrm{CABG}=$ coronary artery bypass graft, $\mathrm{CAD}=$ coronary artery disease, $\mathrm{CHF}=$ congestive heart failure, $\mathrm{COPD}=$ chronic obstructive pulmonary disease, CRUSADE = Can Rapid Risk Stratification of Unstable Angina Patients Suppress Adverse Outcomes with Early Implementation of the American College of Cardiology/American Heart Association Guidelines, DAPT = dual antiplatelet therapy, DES = drug-eluting stent, HBR = high bleeding risk, eGFR = estimated glomerular filtration rate, LVEF = left ventricle ejection fraction, MI = myocardial infarction, NSTEMI = non-ST segment elevation myocardial infarction, $\mathrm{PAD}=$ periphral artery disease, $\mathrm{PARIS}=$ Patterns of Nonadherence to Antiplatelet Regimen In Stented Patients, $\mathrm{PCI}=$ percutaneous coronary intervention, $\mathrm{PPI}=$ proton pump inhibitor, PRAISE = Predicting with Artificial Intelligence Risk after Acute Coronary Syndromes, PRECISE-DAPT = Predicting Bleeding Complications in Patients Undergoing Stent Implantation and Subsequent Dual Antiplatelet Therapy, RCT = randomized controlled trial, REACH = Reduction of Atherothrombosis for Continued Health Registry, STEMI = ST-segment elevation myocardial infarction, UA = unstable angina.

*DAPT score is available at https://tools.acc.org/daptriskapp/\#!/content/calculator/, PRECISE-DAPT is available at http://precisedaptscore.com/predapt/ and PRAISE is available at https://praise.hpc4ai.it/.

bleeding events when a potent $P 2 Y_{12}$ inhibitor was part of the DAPT regime. ${ }^{48}$ Nonetheless, $\mathrm{P}_{2} \mathrm{Y}_{12}$ inhibitor monotherapy is still not widely used in the management of patients after $\mathrm{PCl}$.

\section{What are the indications for dual antiplatelet therapy in patients with atrial fibrillation?}

Dual antiplatelet therapy does not prevent stroke and systemic thromboembolism in patients with atrial fibrillation as effectively as oral anticoagulation..$^{49}$ Conversely, an oral anticoagulant does not reduce coronary ischemic events, including stent thrombosis, as effectively as DAPT. Thus, triple therapy combining DAPT and $\mathrm{OAC}$ is recommended in patients with acute coronary syndromes, either medically managed or after $\mathrm{PCl}$, who also have atrial fibrillation. Direct oral anticoagulants are associated with a lower rate of bleeding events than vitamin $\mathrm{K}$ antagonists, and are therefore preferred in patients with atrial fibrillation, ${ }^{50}$ except in those with mechanical heart valves, moderate-to-severe mitral stenosis or advanced renal disease. Clopidogrel is the $P 2 Y_{12}$ of choice in patients receiving triple therapy, rather than ticagrelor or prasugrel, because of the lower risk of bleeding. ${ }^{3}$

The duration of triple therapy remains a matter of debate, given the increased risk of major bleeding over time. The AUGUSTUS (Open-label, $2 \times 2$ Factorial, Randomized Controlled, Clinical Trial to Evaluate the Safety of Apixaban vs Vitamin K Antagonist and Aspirin vs. Aspirin Placebo in Patients With Atrial Fibrillation and Acute Coronary Syndrome or Percutaneous Coronary Intervention) trial showed that, beyond the first 30 days (the period of 
Table 3: Management of bleeding events in patients with acute coronary syndrome receiving antithrombotic therapy

\section{Antithrombotic treatment modification}

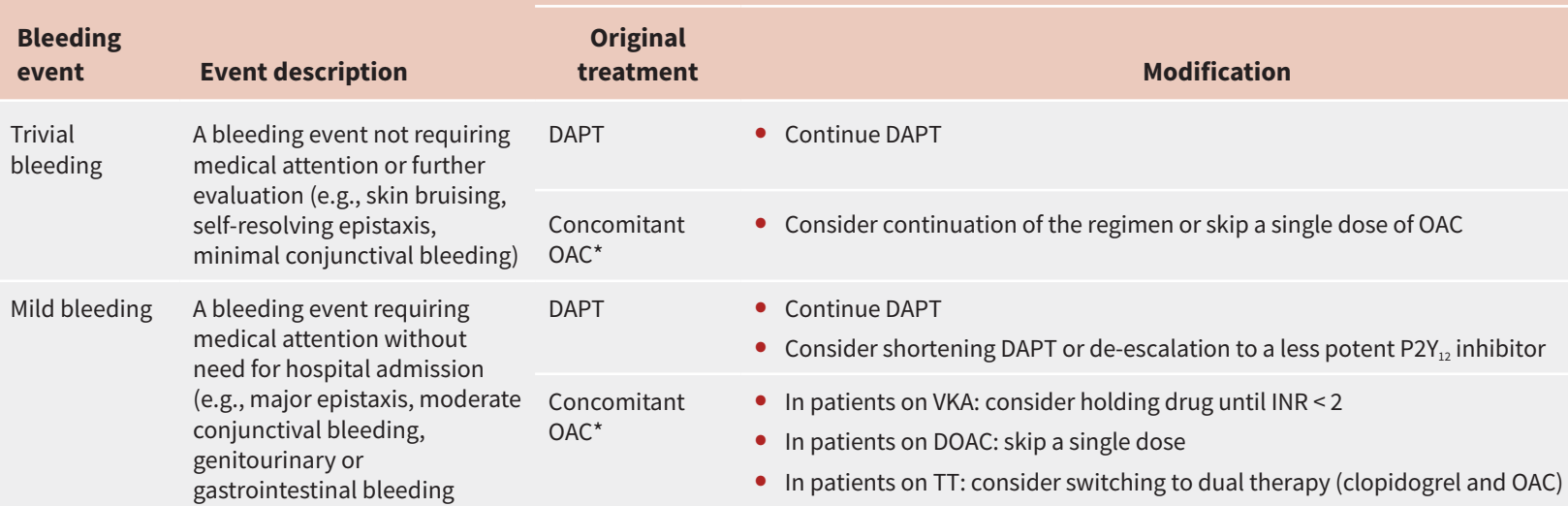

Moderate Ableeding event requiring

bleeding hospital admission or associated with substantial blood loss ( $\geq 3 \mathrm{mmol} / \mathrm{L}$ hemoglobin) without hemodynamic instability (e.g., genitourinary, respiratory, upper or lower gastrointestinal bleeding with substantial blood loss or requiring transfusion)

DAPT

Concomitant $\mathrm{OAC}^{*}$

\section{Severe} bleeding

Lifethreatening bleeding
A bleeding event associated

DAPT ( $\geq 5 \mathrm{mmol} / \mathrm{L}$ hemoglobin) in a hemodynamically unstable patient requiring hospital admission (e.g., severe genitourinary, respiratory or gastrointestinal bleeding, bleeding into critical spaces such as pericardium, retroperitoneum, intraocular spinal or intracranial spaces) OAC*
Any severe active bleeding that poses a threat to a patient's life (e.g., massive genitourinary, respiratory or gastrointestinal bleeding, active intracranial, spinal or intraocular hemorrhage, any bleeding causing hemodynamic instability)
DAPT

Concomitant $\mathrm{OAC}^{*}$
- Consider stopping DAPT and continuing with a single $\mathrm{P}_{2} \mathrm{Y}_{12}$ inhibitor

- Resume DAPT within 3 days if considered safe to do so

- Consider shortening DAPT or de-escalation to a less potent $\mathrm{P}_{2} \mathrm{Y}_{12}$ inhibitor

- Consider stopping OAC or reversing VKA with vitamin $\mathrm{K}$ (unless $\mathrm{CHA}_{2} \mathrm{DS}_{2}-\mathrm{VASC} \geq 4$ or a cardiac assist device or mechanical heart valve is present)

- If DOAC was taken within 2-4 hours, charcoal or dialysis (for patients on dabigatrtan) can be used

- Consider resuming treatment within 1 week, if patient is clinically stable

- In patients on VKA: consider a target INR 2.0-2.5 (unless mechanical heart valve or cardiac assist device is present)

- In patients on DOAC: consider the lowest effective dose

- In patients on TT: consider switching to dual therapy (clopidogrel and OAC)

- Consider stopping DAPT and continue with SAPT (preferably with P2Y ${ }_{12}$ inhibitor)

- Consider stopping all antithrombotic agents if bleeding persists

- Once bleeding has ceased, reassess the need for DAPT or SAPT; if DAPT is resumed, consider shortening length of treatment or de-escalating to a less potent $\mathrm{P} 2 \mathrm{Y}_{12}$ inhibitor

Concomitant - Stop or reverse OAC until bleeding stops (except for patients with an extreme thrombotic risk, i.e., with a mechanical heart valve in mitral position or cardiac assist device)

- In patients on VKA: administer FFP or 4F-PCC

- In patients on DOAC: administer 4F-PPC

- In patients on dabigatran: consider administering idarucizumab

- Consider resuming treatment within 1 week, if clinically stable

- In patients on VKA: consider target INR of 2.0-2.5 (except for patients with mechanical heart valves and cardiac assist devices)

- In patients on DOAC: consider the lowest effective dose

- In patients on TT: consider switching to dual therapy (clopidogrel and OAC)

- Stop all antithrombotic agents immediately

- Once bleeding has ceased, reassess the need for DAPT or SAPT, preferably with clopidogrel (particularly in patients who had upper GI bleeding)

- Stop and reverse OAC

- In patients on VKA: use FFP or 4F-PCC

- In patients on dabigatran: consider administering idarucizumab

Note: $4 \mathrm{~F}-\mathrm{PCC}=4$-factor prothrombin complex concentrate, $\mathrm{ACS}=$ acute coronary syndrome, $\mathrm{CHA}_{2} \mathrm{DS}_{2}-\mathrm{VASC}=$ score that evaluates risk of ischemic stroke, $\mathrm{DAPT}=$ dual antiplatelet therapy, DOAC = direct oral anticoagulant, FFP = fresh frozen plasma, $\mathrm{GI}=$ gastrointestinal, INR = international normalized ratio, $\mathrm{OAC}=$ oral anticoagulant, $\mathrm{SAPT}=$ single antiplatelet therapy, TT = triple therapy, VKA = vitamin $\mathrm{K}$ antagonist.

${ }^{*}$ Concomitant OAC with antiplatelet therapy, including both SAPT and DAPT. 
highest risk for stent thrombosis), ASA and oral anticoagulation increased bleeding events without significantly reducing ischemic events when compared with placebo and oral anticoagulation in patients receiving $\mathrm{P} 2 \mathrm{Y}_{12}$ inhibitors. ${ }^{11,52}$

Numerous studies, including a recent meta-analysis, found that combining oral anticoagulation and single antiplatelet therapy reduced bleeding risk compared with triple therapy. ${ }^{53}$ Importantly, these studies were not powered to detect potential differences in ischemic events, and the meta-analysis found an increased risk of stent thrombosis associated with the combination therapy. ${ }^{53} \mathrm{~A}$ small study of consecutive patients suggested that the ischemic risk was greater in patients who underwent complex $\mathrm{PCl}$ procedures without the use of ASA immediately after a $\mathrm{PCl}$ procedure. ${ }^{54}$ Therefore, triple therapy should be considered in patients at high risk of ischemia and of stent thrombosis and low risk of bleeding for up to 1 month after $\mathrm{PCl}$ (Figure 2). After 1 month, ASA should be stopped, and oral anticoagulation and a $\mathrm{P} 2 \mathrm{Y}_{12}$ inhibitor (preferably clopidogrel, given its lower bleeding risk) should be continued up to 12 months in patients after acute coronary syndrome. At 1 year, oral anticoagulation monotherapy should be used for secondary prevention of stroke. ${ }^{55}$

\section{How should antiplatelet agents be switched?}

De-escalation from a more potent $\mathrm{P} 2 \mathrm{Y}_{12}$ inhibitor to clopidogrel occurs in up to $28 \%$ of patients with acute coronary syndromes, most often because of bleeding or high risk of bleeding. ${ }^{56}$ Similarly, switching between potent $\mathrm{P}_{2} \mathrm{Y}_{12}$ inhibitors may be required if specific adverse effects such as shortness of breath or gout develop in patients receiving ticagrelor. An international consensus document and Canadian guidelines provide guidance to physicians when switching between $\mathrm{P} 2 \mathrm{Y}_{12}$ inhibitors (Box 5). ${ }^{30,57}$

Guided de-escalation therapy by either platelet function testing or CYP2C19-directed genotyping may also be considered in select patients with acute coronary syndromes. ${ }^{58}$

\section{Box 5: Switching between oral $\mathrm{P}_{2} \mathrm{Y}_{12}$ inhibitors \\ Ticagrelor to clopidogrel}

Ticagrelor has a relatively fast offset of action. Clopidogrel should be administered 24 hours after the last dose of ticagrelor. A $600 \mathrm{mg}$ loading dose of clopidogrel should be considered unless the patient had a recent bleeding event, in which case clopidogrel $75 \mathrm{mg}$ should be considered.

\section{Prasugrel to clopidogrel}

The prolonged offset of prasugrel means that the usual clopidogrel maintenance dose of $75 \mathrm{mg}$ daily should be started 24 hours after the last dose of prasugrel.

\section{Ticagrelor to prasugrel}

A $60 \mathrm{mg}$ loading dose of prasugrel should be administered 24 hours after the last dose of ticagrelor.

\section{Prasugrel to ticagrelor}

A $90 \mathrm{mg}$ maintenance dose of ticagrelor should be administered twice daily 24 hours after the last prasugrel dose. If it has been fewer than 30 days since the patient's $\mathrm{PCl}$, a loading dose of $180 \mathrm{mg}$ ticagrelor should be considered.

\section{AF in patients with ACS and an indication for DOAC}

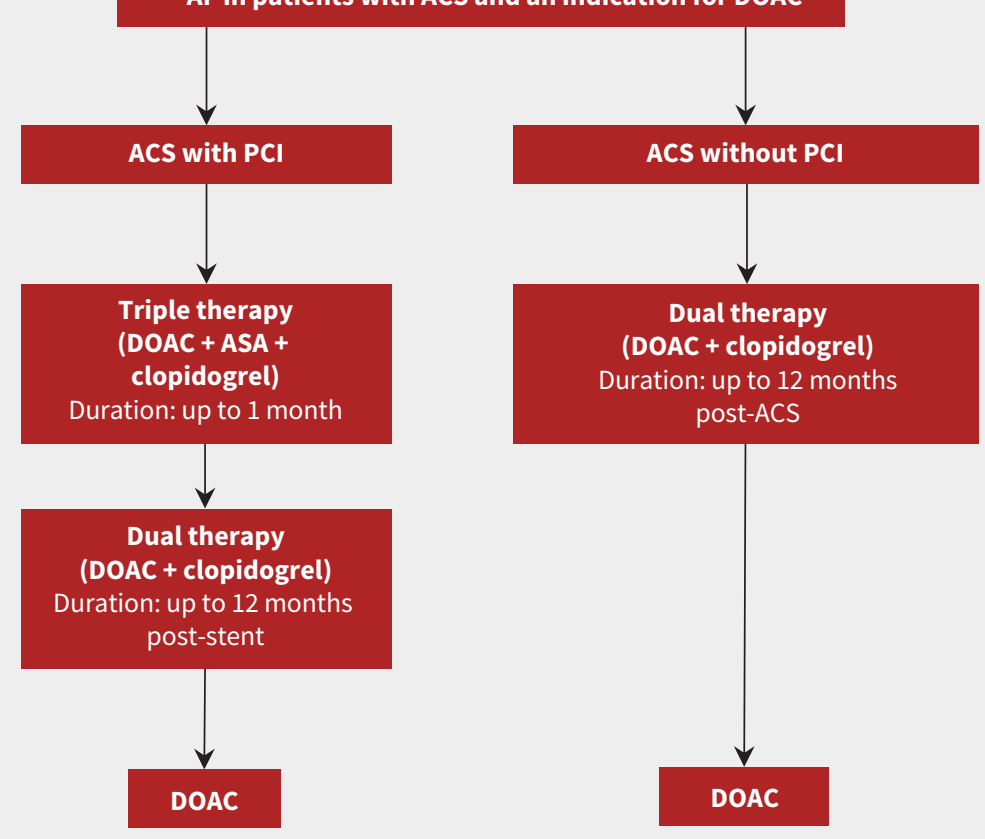

Figure 2: Antiplatelet management in patients with acute coronary syndrome (ACS) and atrial fibrillation (AF). Direct oral anticoagulation (DOAC) is preferred over warfarin; however, if warfarin is to be used the recommended international normalized ratio target is 2.0-2.5. The timing of when to discontinue acetylsalicyclic acid (ASA) will depend on the individual patient's ischemic and bleeding risk. Note: $\mathrm{PCl}=$ percutaneous coronary intervention. 


\section{Conclusion}

Prasugrel and ticagrelor are antiplatelet agents that are more effective than clopidogrel at decreasing the future risk of ischemic events in patients with acute coronary syndromes, but are more likely to cause bleeding. The choice of antiplatelet regimen is influenced by the ischemic and bleeding risk of each patient (Figure 3) . Up to a month of triple therapy with ASA, clopidogrel and an oral anticoagulant should be considered in patients with acute coronary syndromes who also have atrial fibrillation.

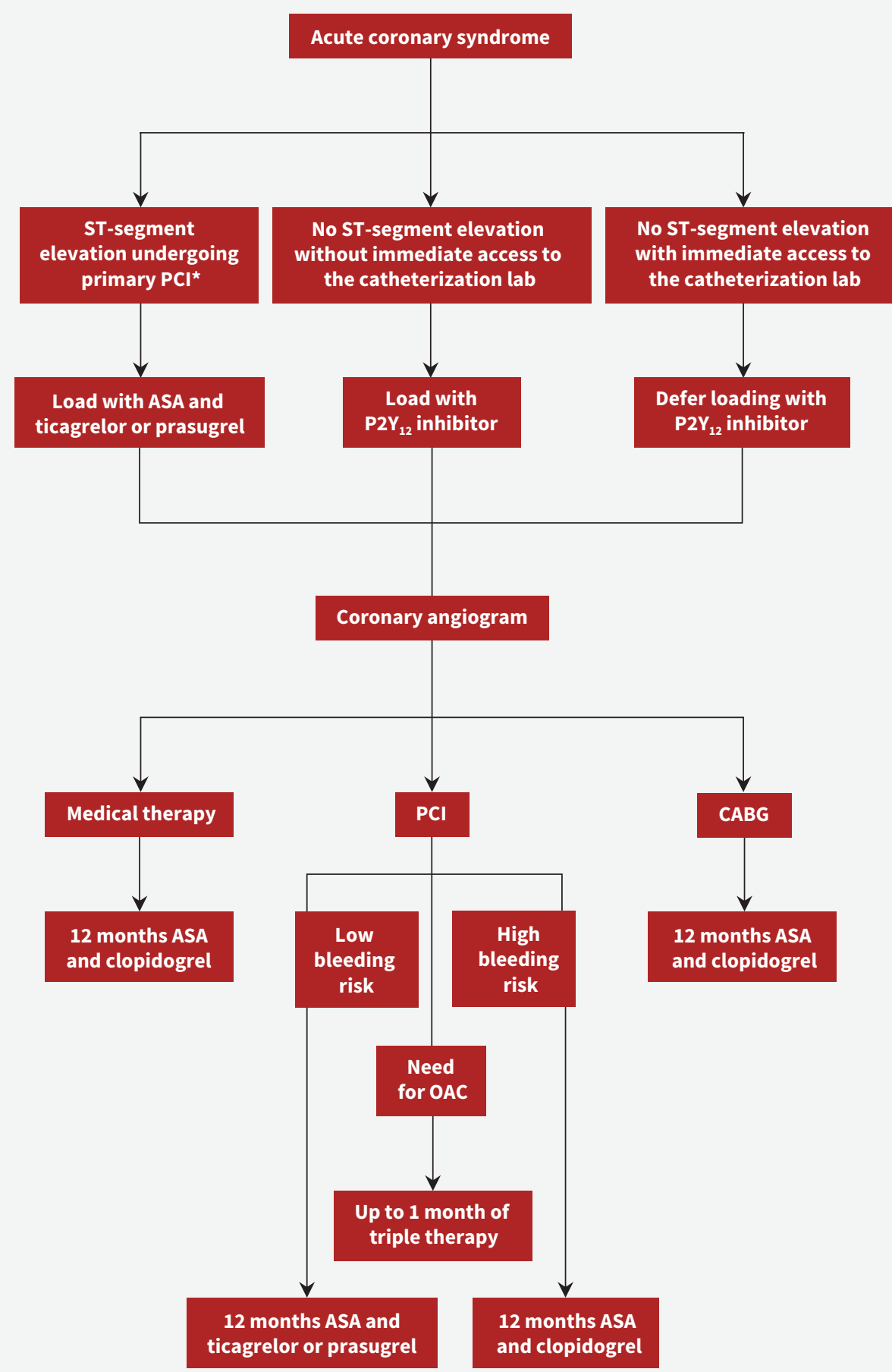

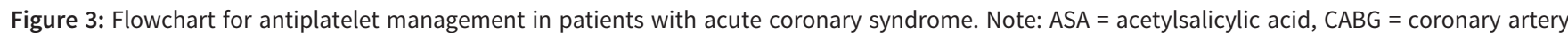
bypass grafting, $\mathrm{OAC}=$ oral anticoagulant, $\mathrm{PCI}=$ percutaneous coronary intervention. ${ }^{*}$ Patients receiving fibrinolytic therapy should be loaded with $\mathrm{ASA}$ and clopidogrel. Switching to ticagrelor within 24 hours should be considered. 


\section{References}

1. Alkhalil M. Mechanistic insights to target atherosclerosis residual risk. Curr Probl Cardiol 2021;46:100432.

2. Ibanez B, James S, Agewall S, et al.; ESC Scientific Document Group. 2017 ESC guidelines for the management of acute myocardial infarction in patients presenting with ST-segment elevation: The Task Force for the management of acute myocardial infarction in patients presenting with ST-segment elevation of the European Society of Cardiology (ESC). Eur Heart J 2018;39:119-77.

3. Collet J-P, Thiele H, Barbato E, et al.; ESC Scientific Document Group. 2020 ESC Guidelines for the management of acute coronary syndromes in patients presenting without persistent ST-segment elevation. Eur Heart J 2021;42: 1289-367.

4. Lewis HD Jr, Davis JW, Archibald DG, et al. Protective effects of aspirin against acute myocardial infarction and death in men with unstable angina. Results of a Veterans Administration Cooperative Study. N Engl J Med 1983;309:396-403.

5. Kuliczkowski W, Witkowski A, Polonski L, et al. Interindividual variability in the response to oral antiplatelet drugs: a position paper of the Working Group on antiplatelet drugs resistance appointed by the Section of Cardiovascular Interventions of the Polish Cardiac Society, endorsed by the Working Group on Thrombosis of the European Society of Cardiology. Eur Heart J 2009;30:426-35.

6. Randomised trial of intravenous streptokinase, oral aspirin, both, or neither among 17,187 cases of suspected acute myocardial infarction: ISIS-2. ISIS-2 (Second International Study of Infarct Survival) Collaborative Group. Lancet 1988;2:349-60.

7. Yusuf S, Zhao F, Mehta SR, et al.; Clopidogrel in Unstable Angina to Prevent Recurrent Events Trial Investigators. Effects of clopidogrel in addition to aspirin in patients with acute coronary syndromes without ST-segment elevation. N Engl J Med 2001;345:494-502.

8. Marquis-Gravel G, Mehta SR, Valgimigli M, et al. A critical comparison of Canadian and international guidelines recommendations for antiplatelet therapy in coronary artery disease. Can J Cardiol 2020;36:1298-307.

9. Bertrand ME, Rupprecht HJ, Urban P, et al.; CLASSIC Investigators. Doubleblind study of the safety of clopidogrel with and without a loading dose in combination with aspirin compared with ticlopidine in combination with aspirin after coronary stenting: the clopidogrel aspirin stent international cooperative study (CLASSICS). Circulation 2000;102:624-9.

10. Chen ZM, Jiang LX, Chen YP, et al.; COMMIT (ClOpidogrel and Metoprolol in Myocardial Infarction Trial) collaborative group. Addition of clopidogrel to aspirin in 45,852 patients with acute myocardial infarction: randomised placebo-controlled trial. Lancet 2005;366:1607-21.

11. Sabatine MS, Cannon CP, Gibson CM, et al.; CLARITY-TIMI 28 Investigators. Addition of clopidogrel to aspirin and fibrinolytic therapy for myocardial infarction with ST-segment elevation. N Engl J Med 2005;352:1179-89.

12. Wallentin L, Becker RC, Budaj A, et al. Ticagrelor versus clopidogrel in patients with acute coronary syndromes. N Engl J Med 2009;361:1045-57.

13. Wiviott SD, Braunwald E, McCabe CH, et al.; TRITON-TIMI 38 Investigators. Prasugrel versus clopidogrel in patients with acute coronary syndromes. N Engl J Med 2007;357:2001-15.

14. Schüpke S, Neumann F-J, Menichelli M, et al.; ISAR-REACT 5 Trial Investigators. Ticagrelor or prasugrel in patients with acute coronary syndromes. N Engl J Med 2019;381:1524-34.

15. Crea F, Thiele H, Sibbing D, et al. Debate: prasugrel rather than ticagrelor is the preferred treatment for NSTE-ACS patients who proceed to $\mathrm{PCI}$ and pretreatment should not be performed in patients planned for an early invasive strategy. Eur Heart J 2021;42:2973-85.

16. Navarese EP, Khan SU, Kolodziejczak M, et al. Comparative efficacy and safety of oral $P 2 Y_{12}$ inhibitors in acute coronary syndrome: network meta-analysis of 52816 patients from 12 randomized trials. Circulation 2020;142:150-60.

17. Berwanger O, Lopes RD, Moia DDF, et al. Ticagrelor versus clopidogrel in patients with STEMI treated with fibrinolysis: TREAT trial. J Am Coll Cardiol 2019;73:2819-28.

18. Valgimigli M, Bueno H, Byrne RA, et al.; ESC Scientific Document Group; ESC Committee for Practice Guidelines (CPG); ESC National Cardiac Societies. 2017 ESC focused update on dual antiplatelet therapy in coronary artery disease developed in collaboration with EACTS: the Task Force for dual antiplatelet therapy in coronary artery disease of the European Society of Cardiology (ESC) and of the European Association for Cardio-Thoracic Surgery (EACTS). Eur Heart J 2018;39:213-60.

19. Lindholm D, Varenhorst C, Cannon CP, et al. Ticagrelor vs. clopidogrel in patients with non-ST-elevation acute coronary syndrome with or without revascularization: results from the PLATO trial. Eur Heart J 2014;35:2083-93.

20. Montalescot G, Bolognese L, Dudek D, et al.; ACCOAST Investigators. Pretreatment with prasugrel in non-ST-segment elevation acute coronary syndromes. N Engl J Med 2013;369:999-1010.
21. Tarantini G, Mojoli M, Varbella F, et al.; DUBIUS Investigators; Italian Society of Interventional Cardiology. Timing of oral $\mathrm{P}_{2} \mathrm{Y}_{12}$ inhibitor administration in nonST elevation acute coronary syndrome. J Am Coll Cardiol 2020;76:2450-9.

22. Bonaca MP, Bhatt DL, Coeh M, et al.; PEGASUS-TIMI 54 Steering Committee and Investigators. Long-term use of ticagrelor in patients with prior myocardial infarction. N Engl J Med 2015;372:1791-800.

23. Mauri L, Kereiakes DJ, Yeh RW, et al.; DAPT Study Investigators. Twelve or 30 months of dual antiplatelet therapy after drug-eluting stents. N Engl J Med 2014;371:2155-66.

24. Khan SU, Singh M, Valavoor S, et al. Dual antiplatelet therapy after percutaneous coronary intervention and drug-eluting stents: a systematic review and network meta-analysis. Circulation 2020;142:1425-36.

25. Hahn J-Y, Song YB, Oh J-H, et al.; SMART-DATE investigators. 6-month versus 12-month or longer dual antiplatelet therapy after percutaneous coronary intervention in patients with acute coronary syndrome (SMART-DATE): a randomised, open-label, non-inferiority trial. Lancet 2018;391:1274-84.

26. Valgimigli M, Frigoli E, Heg D, et al.; MASTER DAPT Investigators. Dual antiplatelet therapy after $\mathrm{PCl}$ in patients at high bleeding risk. $N$ Engl J Med 2021;385:1643-55.

27. Koo B-K, Kang J, Park KW, et al.; HOST-EXAM investigators. Aspirin versus clopidogrel for chronic maintenance monotherapy after percutaneous coronary intervention (HOST-EXAM): an investigator-initiated, prospective, randomised, open-label, multicentre trial. Lancet 2021;397:2487-96.

28. Eikelboom JW, Connolly SJ, Bosch J, et al.; COMPASS Investigators. Rivaroxaban with or without aspirin in stable cardiovascular disease. N Engl J Med 2017;377:1319-30.

29. Valgimigli M, Bueno H, Byrne RA, et al.; ESC Scientific Document Group; ESC Committee for Practice Guidelines (CPG); ESC National Cardiac Societies. ESC focused update on dual antiplatelet therapy in coronary artery disease developed in collaboration with EACTS: The Task Force for dual antiplatelet therapy in coronary artery disease of the European Society of Cardiology (ESC) and of the European Association for Cardio-Thoracic Surgery (EACTS). Eur Heart $J$ 2018;39:213-60.

30. Subherwal S, Bach RG, Chen AY, et al. Baseline risk of major bleeding in nonST-segment-elevation myocardial infarction: the CRUSADE (Can Rapid risk stratification of Unstable angina patients Suppress ADverse outcomes with Early implementation of the ACC/AHA guidelines) bleeding score. Circulation 2009;119:1873-82.

31. Mehran R, Pocock SJ, Nikolsky E, et al. A risk score to predict bleeding in patients with acute coronary syndromes. J Am Coll Cardiol 2010;55:2556-66.

32. Ducrocq G, Wallace JS, Baron G, et al.; REACH Investigators. Risk score to predict serious bleeding in stable outpatients with or at risk of atherothrombosis. Eur Heart J 2010;31:1257-65.

33. Yeh RW, Secemsky EA, Kereiakes DJ, et al.; DAPT Study Investigators. Development and validation of a prediction rule for benefit and harm of dual antiplatelet therapy beyond 1 year after percutaneous coronary intervention. JAMA 2016;315:1735-49.

34. Baber U, Mehran R, Giustino G, et al. Coronary thrombosis and major bleeding after $\mathrm{PCl}$ with drug-eluting stents: risk scores from PARIS. J Am Coll Cardiol 2016;67:2224-34.

35. Costa F, van Klaveren D, James S, et al.; PRECISE-DAPT Study Investigators. Derivation and validation of the predicting bleeding complications in patients undergoing stent implantation and subsequent dual antiplatelet therapy (PRECISE-DAPT) score: a pooled analysis of individual-patient datasets from clinical trials. Lancet 2017;389:1025-34.

36. Urban P, Gregson J, Owen R, et al. Assessing the risks of bleeding vs thrombotic events in patients at high bleeding risk after coronary stent implantation: the ARC-high bleeding risk trade-off model. JAMA Cardiol 2021;6:410-9.

37. Urban P, Mehran R, Colleran R, et al. Defining high bleeding risk in patients undergoing percutaneous coronary intervention: a consensus document from the Academic Research Consortium for High Bleeding Risk. Eur Heart $J$ 2019;40:2632-53.

38. D'Ascenzo F, De Filippo O, Gallone G, et al.; PRAISE study group. Machine learning-based prediction of adverse events following an acute coronary syndrome (PRAISE): a modelling study of pooled datasets. Lancet 2021;397:199-207.

39. Mihatov N, Secemsky EA, Kereiakes DJ, et al. Utility of the dual antiplatelet therapy score to guide antiplatelet therapy: a systematic review and metaanalysis. Catheter Cardiovasc Interv 2021;97:569-78.

40. Choi SY, Kim MH, Cho Y-R, et al. Performance of PRECISE-DAPT score for predicting bleeding complication during dual antiplatelet therapy. Circ Cardiovasc Interv 2018;11:e006837.

41. Capodanno D, Bhatt DL, Gibson CM, et al. Bleeding avoidance strategies in percutaneous coronary intervention. Nat Rev Cardiol 2022;19:117-32. 
42. Windecker S, Latib A, Kedhi E, et al.; ONYX ONE Investigators. Polymer-based or polymer-free stents in patients at high bleeding risk. N Engl J Med 2020; 382:1208-18.

43. Wärme J, Sundqvist M, Mars K, et al. Helicobacter pylori screening in clinical routine during hospitalization for acute myocardial infarction. Am Heart $J$ 2021;231:105-9.

44. Gimbel ME, Minderhoud SCS, Ten Berg JM. A practical guide on how to handle patients with bleeding events while on oral antithrombotic treatment. Neth Heart J 2018;26:341-51.

45. Bhatt DL, Pollack CV, Weitz JI, et al. Antibody-based ticagrelor reversal agent in healthy volunteers. N Engl J Med 2019;380:1825-33.

46. Baber U, Zafar MU, Dangas G, et al. Ticagrelor with or without aspirin after $\mathrm{PCl}$ : the TWILIGHT platelet substudy. J Am Coll Cardiol 2020;75:578-86.

47. O'Donoghue ML, Murphy SA, Sabatine MS. The safety and efficacy of aspirin discontinuation on a background of a $\mathrm{P} 2 \mathrm{Y}_{12}$ inhibitor in patients after percutaneous coronary intervention: a systematic review and meta-analysis. Circulation 2020;142:538-45.

48. Valgimigli M, Gragnano F, Branca M, et al. $\mathrm{P}_{2} \mathrm{Y}_{12}$ inhibitor monotherapy or dual antiplatelet therapy after coronary revascularisation: individual patient level meta-analysis of randomised controlled trials. BMJ 2021;373.

49. Lip GYH, Collet J-P, Haude M, et al. Management of antithrombotic therapy in $\mathrm{AF}$ patients presenting with $\mathrm{ACS}$ and/or undergoing $\mathrm{PCl}$ : a summary of the joint consensus document of the European Heart Rhythm Association (EHRA), European Society of Cardiology Working Group on Thrombosis, European Association of Percutaneous Cardiovascular Interventions (EAPCI) and European Association of Acute Cardiac Care (ACCA) endorsed by the Heart Rhythm Society (HRS), Asia-Pacific Heart Rhythm Society (APHRS), Latin America Heart Rhythm Society (LAHRS), and Cardiac Arrhythmia Society of Southern Africa (CASSA). Eur Heart J 2018;39:2847-50.

50. Lopes RD, Heizer G, Aronson R, et al.; AUGUSTUS Investigators. Antithrombotic therapy after acute coronary syndrome or $\mathrm{PCl}$ in atrial fibrillation. $\mathrm{N}$ Engl $\mathrm{J}$ Med 2019;380:1509-24.
51. Lopes RD, Leonardi S, Wojdyla DM, et al. Stent thrombosis in patients with atrial fibrillation undergoing coronary stenting in the AUGUSTUS trial. Circulation 2020;141:781-3.

52. Alexander JH, Wojdyla D, Vora AN, et al. Risk/benefit tradeoff of antithrombotic therapy in patients with atrial fibrillation early and late after an acute coronary syndrome or percutaneous coronary intervention: insights from AUGUSTUS. Circulation 2020;141:1618-27.

53. Gargiulo G, Goette A, Tijssen J, et al. Safety and efficacy outcomes of double vs. triple antithrombotic therapy in patients with atrial fibrillation following percutaneous coronary intervention: a systematic review and meta-analysis of non-vitamin $\mathrm{K}$ antagonist oral anticoagulant-based randomized clinical trials. Eur Heart J 2019;40:3757-67.

54. Alkhalil M, Shahmohammadi M, Spence MS, et al. Aspirin discontinuation in patients requiring oral anticoagulation undergoing percutaneous coronary intervention, the role of procedural complexity. Cardiovasc Drugs Ther 2020; 34:659-62.

55. Yasuda S, Kaikita K, Akao M, et al.; AFIRE Investigators. Antithrombotic therapy for atrial fibrillation with stable coronary disease. N Engl J Med 2019;381: 1103-13.

56. Zettler ME, Peterson ED, McCoy LA, et al.; TRANSLATE-ACS Investigators. Switching of adenosine diphosphate receptor inhibitor after hospital discharge among myocardial infarction patients: Insights from the Treatment with Adenosine Diphosphate Receptor Inhibitors: Longitudinal Assessment of Treatment Patterns and Events after Acute Coronary Syndrome (TRANSLATEACS) observational study. Am Heart J 2017;183:62-8.

57. Angiolillo DJ, Rollini F, Storey RF, et al. International expert consensus on switching platelet $\mathrm{P} 2 \mathrm{Y}_{12}$ receptor-inhibiting therapies. Circulation 2017; 136:1955-75.

58. Sibbing D, Aradi D, Alexopoulos D, et al. Updated expert consensus statement on platelet function and genetic testing for guiding $P 2 Y_{12}$ receptor inhibitor treatment in percutaneous coronary intervention. JACC Cardiovasc Interv 2019;12:1521-37.
Competing interests: Alan Bell reports consulting fees from AstraZeneca, Bayer and Sanofi; speaker fees from AstraZeneca; and board membership with Thrombosis Canada, Hypertension Canada and the Canadian Cardiovascular Society. Sol Stern reports honoraria from Bayer, Pfizer, Bristol Myers Squibb and Sea Courses Inc. Shaun Goodman reports research grant support from Amgen, Anthos Therapeutics, AstraZeneca, Bayer, Boehringer Ingelheim, Bristol Myers Squibb, CSL Behring, Eli Lilly, Esperion, Ferring Pharmaceuticals, Merck, Novartis, Pfizer, Regeneron, Sanofi, Heart and Stroke Foundation of Ontario, Canadian Heart Research Centre and MD Primer, Canadian VIGOUR Centre, Cleveland Clinic Coordinating Center for Clinical Research, Duke Clinical Research Institute, New York University Clinical Coordinating Center, PERFUSE Research Institute and TIMI Study Group (Brigham Health). He also reports consulting honoraria from Amgen, AstraZeneca, Bayer, Boehringer Ingelheim, Bristol Myers Squibb, CSL Behring, Eli Lilly, Ferring Pharmaceuticals, HLS Therapeutics, JAMP Pharma, Merck, Novartis, PendoPharm of Pharmascience, Pfizer, Regeneron, Sanofi, Servier, Valeo Pharma, Canadian Heart Research Centre and MD Primer, and speaking fees from Amgen, AstraZeneca, Bayer, Boehringer Ingelheim, Bristol Myers Squibb, Eli Lilly, Ferring Pharmaceuticals, HLS Therapeutics, JAMP Pharma, Novartis, PendoPharm of Pharmascience, Pfizer, Regeneron, Sanofi, Servier, Valeo Pharm, Canadian Heart Research Centre and MD Primer. He sits on boards with American Regent of Daiichi-Sankyo and Novo Nordisk and is co-director of the Canadian VIGOUR Centre. All competing interests are outside the submitted work. No other competing interests were declared.

This article has been peer reviewed.
Affiliations: Division of Cardiology (Alkhalil, Kuzemczak), Toronto General Hospital, Toronto, Ont.; Cardiothroacic Centre, Freeman Hospital (Alkhalil); Translational and Clinical Research Institute (Alkhalil), Newcastle University, Newcastle upon Tyne, UK; Division of Emergency Medicine (Kuzemczak), Poznan University of Medical Sciences, Poznań, Poland; Department of Interventional Cardiology (Kuzemczak), Central Clinical Hospital of the Ministry of Interior and Administration, Warsaw, Poland; Department of Family and Community Medicine (Bell), University of Toronto, Toronto, Ont.; Argus Medical Centre Family Health Organization (Stern), Oakville, Ont.; Division of Emergency Medicine (Welsford), McMaster University, Hamilton, Ont.; Division of Cardiology (Cantor), University of Toronto and Southlake Regional Health Centre; Division of Cardiology (Goodman), St. Michael's Hospital, Department of Medicine, University of Toronto, Toronto, Ont.

Contributors: All of the authors contributed to the conception and design of the work, drafted the manuscript, revised it critically for important intellectual content, gave final approval of the version to be published and agreed to be accountable for all aspects of the work.

Content licence: This is an Open Access article distributed in accordance with the terms of the Creative Commons Attribution (CC BY-NC-ND 4.0) licence, which permits use, distribution and reproduction in any medium, provided that the original publication is properly cited, the use is noncommercial (i.e., research or educational use), and no modifications or adaptations are made. See: https://creativecommons.org/licenses/ by-nc-nd/4.0/

Correspondence to: Mohammad Alkhalil, mak-83@hotmail.com 